\title{
Review of Vector Condensation at High Chemical Potential
}

\author{
Francesco Sannino \\ NORDITA, Blegdamsvej 17, Copenhagen Ø, DK-2100, Denmark
}

\begin{abstract}
Here I review vectorial type condensation due to a non zero chemical potential associated to some of the global conserved charges of the theory. The phase structure is very rich since three distinct phases exists depending on the value assumed by one of the zero chemical potential vector self interaction terms. In a certain limit of the couplings and for large chemical potential the theory is not stable. This limit corresponds to a gauge type limit often employed to economically describe the ordinary vector mesons self interactions in QCD. Our analysis is relevant since it leads to a number of physical applications not limited to strongly interacting theories at non zero chemical potential.
\end{abstract}

\section{INTRODUCTION}

Relativistic vector condensation has been proposed and studied in different realms of theoretical physics. However the condensation mechanism and the nature of the relativistic vector mesons themselves is quite different. Linde [1, 2, 3], for example, proposed the condensation of the intermediate vector boson $W$ in the presence of a superdense fermionic matter while Ambjørn and Olesen [4, 5] investigated their condensation in presence of a high external magnetic field. Manton [6] and later on Hosotani [7] considered the extension of gauge theories in extra dimensions and suggested that when the extra dimensions are non simply connected the gauge fields might condense. Li in [8] has also explored a simple effective Lagrangian and the effects of vector condensation when the vectors live in extra space dimensions ${ }^{1}$.

In [9, 10] it has also been suggested that the non gauge vectors fields such as the (quark) composite field $\rho$ in QCD may become light and possibly condense in a high quark matter density and/or in hot QCD. Harada and Yamawaki's [11] dynamical computations within the framework of the hidden local gauge [12] symmetry support this picture. Recently the Bose-Einstein relativistic condensation phenomenon has been suggested as physical mechanism underlying the electroweak symmetry breaking scenario [13, 14]

We consider another type of condensation. If vectors themselves carry some global charges we can introduce a non zero chemical potential associated to some of these

\footnotetext{
${ }^{1}$ The effective Lagrangian and the condensation phenomenon in extra (non compact) space dimensions has also been studied/suggested in [16].
} 
charges. If the chemical potential is sufficiently high one can show that the gaps (i.e. the energy at zero momentum) of these vectors become light [15, 16, 17] and eventually zero signaling an instability. If one applies our results to 2 color Quantum Chromo Dynamics (QCD) at non zero baryon chemical potential one predicts that the vectors made out of two quarks (in the 2 color theory the baryonic degrees of freedom are bosons) condense. Recently lattice studies for 2 color at high baryonic potential [18] seem to support our predictions. This is the relativistic vectorial Bose-Einstein condensation phenomenon. A decrease in the gap of vectors is also suggested at high baryon chemical potential for two colors in [19].

I review here the general structure of vector condensation [15].

\section{VACUUM STRUCTURE AND DIFFERENT PHASES}

We choose to consider the following general effective Lagrangian for a relativistic massive vector field in the adjoint of $S U(2)$ in $3+1$ dimensions and up to four vector fields, two derivatives and containing only intrinsic positive parity terms $[20,21]^{2}$ :

$$
\begin{aligned}
L & =-\frac{1}{4} F_{\mu \nu}^{a} F^{a \mu \nu}+\frac{m^{2}}{2} A_{\mu}^{a} A^{a \mu}+\delta \varepsilon^{a b c} \partial_{\mu} A_{a v} A_{b}^{\mu} A_{c}^{v} \\
& -\frac{\lambda}{4}\left(A_{\mu}^{a} A^{a \mu}\right)^{2}+\frac{\lambda^{\prime}}{4}\left(A_{\mu}^{a} A^{a v}\right)^{2},
\end{aligned}
$$

with $F_{\mu \nu}^{a}=\partial_{\mu} A_{v}^{a}-\partial_{v} A_{\mu}^{a}, a=1,2,3$ and metric convention $\eta^{\mu \nu}=\operatorname{diag}(+,-,-,-)$. Here, $\delta$ is a real dimensionless coefficient, $m^{2}$ is the tree level mass term and $\lambda$ and $\lambda^{\prime}$ are positive dimensionless coefficients with $\lambda \geq \lambda^{\prime}$ when $\lambda^{\prime} \geq 0$ or $\lambda \geq 0$ when $\lambda^{\prime} \geq 0$ to insure positivity of the potential. The Lagrangian describes a self interacting $S U(2)$ Yang-Mills theory in the limit $m^{2}=0, \lambda=\lambda^{\prime}>0$ and $\delta=-\sqrt{\lambda}$.

We set $\delta=0$ and the theory gains a new symmetry according to which we have always a total number of even vectors in any process [15]. The effect of a nonzero chemical potential associated to a given conserved charge - (say $T^{3}=\tau^{3} / 2$ ) - can be included by modifying the derivatives acting on the vector fields according to $\partial_{v} A_{\rho} \rightarrow$ $\partial_{v} A_{\rho}-i\left[B_{v}, A_{\rho}\right]$ with $B_{v}=\mu \delta_{v 0} T^{3} \equiv V_{v} T^{3}$ where $V=(\mu, \overrightarrow{0})$. The chemical potential breaks explicitly the Lorentz transformation leaving invariant the rotational symmetry. Also the $S U(2)$ internal symmetry breaks to a $U(1)$ symmetry. If the $\delta$ term is absent we have an extra unbroken $Z_{2}$ symmetry which acts according to $A_{\mu}^{3} \rightarrow-A_{\mu}^{3}$. These symmetries suggest introducing the following cylindric coordinates:

$$
\phi_{\mu}=\frac{1}{\sqrt{2}}\left(A_{\mu}^{1}+i A_{\mu}^{2}\right), \quad \phi_{\mu}^{*}=\frac{1}{\sqrt{2}}\left(A_{\mu}^{1}-i A_{\mu}^{2}\right), \quad \psi_{\mu}=A_{\mu}^{3},
$$

\footnotetext{
${ }^{2}$ For simplicity and in view of the possible physical applications we take the vectors to belong to the adjoint representation of the $S U(2)$ group.
} 
on which the covariant derivative acts as follows:

$$
D_{\mu} \phi_{v}=(\partial+i V)_{\mu} \phi_{v}, \quad D_{\mu} \psi_{v}=\partial_{\mu} \psi_{v}, \quad V_{v}=(\mu, \mathbf{0})
$$

The vacuum structure of the theory is explored via the variational ansatz [15]:

$$
\psi^{\mu}=0, \quad \phi^{\mu}=\sigma\left(\begin{array}{c}
0 \\
1 \\
e^{i \alpha} \\
0
\end{array}\right) \text {. }
$$

Substituting the ansatz in the potential expression yields:

$$
V=2 \sigma^{4}\left[\left(2 \lambda-\lambda^{\prime}\right)-\lambda^{\prime} \cos ^{2} \alpha\right]+2\left(m^{2}-\mu^{2}\right) \sigma^{2}
$$

The potential is positive for any value of $\alpha$ when $\lambda>\lambda^{\prime}$ if $\lambda^{\prime} \geq 0$ or $\lambda>0$ if $\lambda^{\prime}<0$. Due to our ansatz the ground state is independent of $\delta$. The unbroken phase occurs when $\mu \leq m$ and the minimum is at $\sigma=0$. A possible broken phase is achieved when $\mu>m$ since in this case the quadratic term in $\sigma$ is negative. According to the value of $\lambda^{\prime}$ we distinguish three distinct phases:

\section{The polar phase: $\lambda^{\prime}>0$}

In this phase the minimum is for

$$
\left\langle\phi^{\mu}\right\rangle=\sigma\left(\begin{array}{l}
0 \\
1 \\
1 \\
0
\end{array}\right), \quad \text { with } \quad \sigma^{2}=\frac{1}{4} \frac{\mu^{2}-m^{2}}{\lambda-\lambda^{\prime}},
$$

and we have the following pattern of symmetry breaking $S O(3) \times U(1) \rightarrow S O(2)$, where $S O(3)$ is the rotational group. We have three broken generators and 3 gapless excitations with linear dispersion relations. All of the physical states (with and without a gap) are either vectors (2-component) or scalars with respect to the unbroken $S O(2)$ group. The dispersion relations for the 3 gapless states can be found in [15].

At $\mu=m$ the dispersion relations are no longer linear in the momentum. This is related to the fact that the specific part of the potential term has a partial conformal symmetry discussed first in [16]. Some states in the theory are curvatureless but the chemical potential present in the derivative term prevents these states to be gapless. There is a transfer of the conformal symmetry information from the potential term to the vanishing of the velocity of the gapless excitations related to the would be gapless states. This conversion is due to the linear time-derivative term induced by the presence of the chemical potential term [22, 16, 15]. 


\section{Enhanced symmetry and type II Goldstone bosons: $\lambda^{\prime}=0$}

Here the potential has an enhanced $S O(6)$ in contrast to the $S U(2) \times U(1)$ for $\lambda^{\prime} \neq 0$ global symmetry which breaks to an $S O(5)$ with 5 broken generators. Expanding the potential around the vacuum we find 5 null curvatures [15]. However we have only three gapless states obtained diagonalizing the quadratic kinetic term and the potential term. Two states (a vector of $S O(2)$ ) become type II goldstone bosons while the scalar state remains type I [23]. This latter state is the goldstone boson related to the spontaneously broken $U(1)$ symmetry $^{3}$. Using the Nielsen Chadha theorem[23] the type II states are counted twice with respect to the number of broken generators while the linear just once recovering the number of generators broken by the vacuum. We can be more specific since we discovered [16] that the velocity of the associated gapless states is proportional to the curvatures (evaluate on the minimum) of the would be goldstone bosons which is zero in the $\lambda^{\prime}=0$ limit. Again we have an efficient mechanism for communicating the information of the extra broken symmetries from the curvatures to the velocities of the already gapless excitations.

\section{The apolar phase: $\lambda^{\prime}<0$}

In this case the potential is minimized for:

$$
\left\langle\phi^{\mu}\right\rangle=\sigma\left(\begin{array}{l}
0 \\
1 \\
l \\
0
\end{array}\right), \quad \text { with } \quad \sigma^{2}=\frac{1}{2} \frac{\mu^{2}-m^{2}}{2 \lambda-\lambda^{\prime}},
$$

with 3 broken generators. However the unbroken generator is a linear combination of the $U(1)$ and a $S O(3)$ generator and in [15] it has been shown that only two gapless states emerges. One of the two states is a type I goldstone boson while the other is type II. The two goldstone bosons are one in the $z$ and the other in the $x-y$ plane. Interestingly in this phase, due to the intrinsic complex nature of the vev, we have spontaneous $C P$ breaking. We summarize in Fig. 1 the phase structure in terms of the number of goldstone bosons and their type according to the values assumed by $\lambda^{\prime}$.

\section{The case $\lambda=\lambda^{\prime}$ : the gauge theory limit}

Here the potential is:

$$
V=2 \sigma^{4} \lambda \sin ^{2} \alpha+2\left(m^{2}-\mu^{2}\right) \sigma^{2}
$$

\footnotetext{
3 According to the Nielsen and Chadha counting scheme in absence of Lorentz invariance if $n_{I}$ denotes the number of gapless excitations of type $I$ with linear dispersion relations (i.e. $E \propto p$ ) and $n_{I I}$ the ones with quadratic dispersion relations (i.e. $E \propto p^{2}$ ) the Goldstone theorem generalizes to $n_{I}+2 n_{I I} \geq$ \# broken generators.
} 


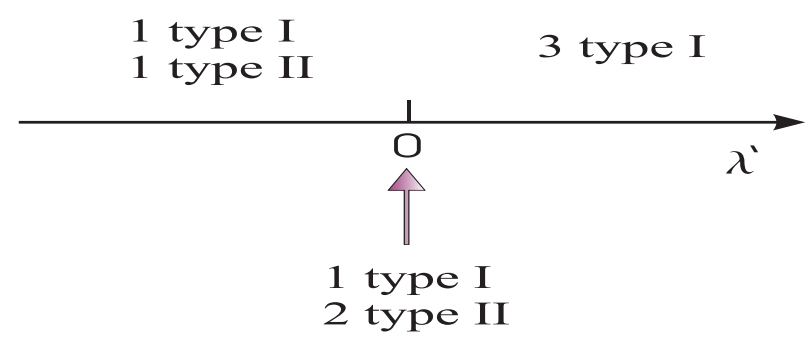

FIGURE 1. We show the number and type of goldstone bosons in the three distinct phases associated to the value assumed by the coupling $\lambda^{\prime}$. In the polar phase, positive $\lambda^{\prime}$, we have 3 type I goldstone bosons. In the apolar phase, negative $\lambda^{\prime}$, we have one type I and one type II goldstone boson while in the enhanced symmetry case $\lambda^{\prime}=0$ we have one type I and two type II excitations.

with two extrema when $\mu>m$, one for $\alpha=0$ and $\sigma=0$ which is an unstable point and the other for $\alpha= \pm \pi / 2$ and $\sigma^{2}=\frac{\left(\mu^{2}-m^{2}\right)}{2 \lambda}$ corresponding to a saddle point (see the potential in Fig. (2). At first the fact that we have no stable solutions seems unreasonable

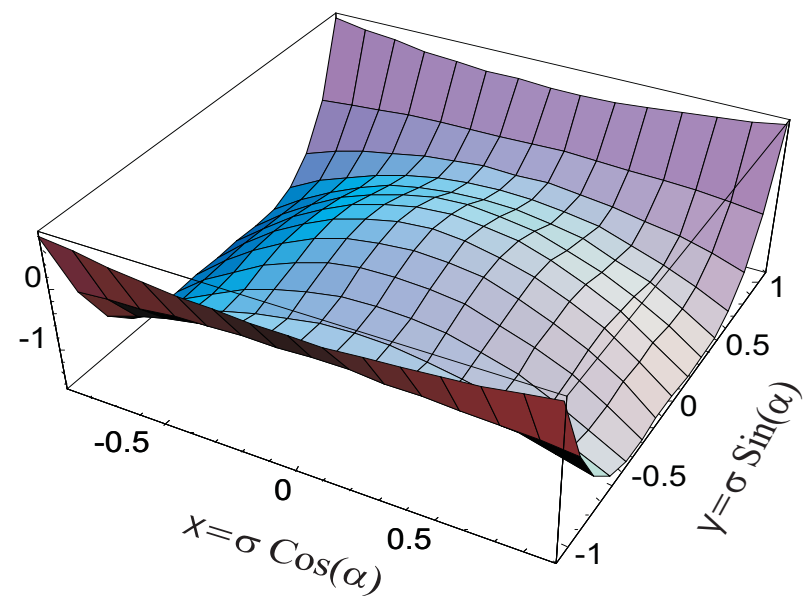

FIGURE 2. Potential plotted for $\mu=2 m$ and $\lambda=\lambda^{\prime}=1$.

since we know that in literature we often encounter condensation of intermediate vector mesons such as the $W$ boson. However (except for extending the theory in higher space dimensions) in these cases one often introduces an external source. For example one adds to the theory a strong magnetic field (say in the direction $z$ ) which couples to the electromagnetically charged intermediate vector bosons $W^{+}$and $W^{-}$). In this case the potential is (see [4]):

$$
V=2 \sigma^{4} \lambda \sin ^{2} \alpha+2\left(m^{2}-e H \sin \alpha\right) \sigma^{2},
$$

where $e$ is the electromagnetic charge and $H$ is the external electromagnetic source field. This potential has a true minimum for $\alpha=\pi / 2$ and $\sigma=\frac{e H-m^{2}}{2 \lambda}$ whenever the external magnetic field satisfies the relation $\mathrm{eH}>m$. We learn that the relativistic vector theory is unstable at large chemical potential whenever the non derivative vector self interactions 
are tuned to be identical. This is precisely the limit often used in literature when writing effective Lagrangians that in QCD describe the $\rho$ vector field. In principle we can still imagine to stabilize the potential in the gauge limit by adding some higher order operators. This might be the case if one introduces massive gauge bosons as in [12]. Due to the gauge limit $\lambda^{\prime}=\lambda$ is positive and assuming the higher order corrections to be small one predicts a polar phase within this model. Another solution to this instability is that the chemical potential actually does not rise above the mass of the vectors even if we increase the relative charge density. This phenomenon is similar to what happens in the case of an ideal bose gas at high chemical potential [24]. If the strict gauge limit is taken (i.e. also the mass term is set to zero) we need to impose gauge neutrality and the analysis modifies [24].

Interestingly by studying the vector condensation phenomenon for strongly interacting theories on the lattice at high isospin chemical potential we can determine the best way of describing the ordinary vector self-interactions at zero chemical potential.

\section{PHYSICAL APPLICATIONS AND CONCLUSIONS}

We presented the phase structure of the relativistic massive vector condensation phenomenon due to a non zero chemical potential associated to some of the global conserved charges of the theory [15]. The phase structure is very rich. According to the value assumed by $\lambda^{\prime}$ we have three independent phases. The polar phase with $\lambda^{\prime}$ positive is characterized by a real vacuum expectation value and 3 goldstone bosons of type I. The apolar phase for $\lambda^{\prime}$ negative has a complex vector vacuum expectation value spontaneously breaking CP. In this phase we have one goldstone boson of type I and one of type II while still breaking 3 continuous symmetries. The third phase has an enhanced potential type symmetry and 3 goldstone bosons one of type I and two of type II.

We also discovered that if we force the self interaction couplings $\lambda$ and $\lambda^{\prime}$ to be identical, as predicted in a Yang-Mills massive theory, our ansatz for the vacuum does not lead to a stable minimum when increasing the chemical potential above the mass of the vectors. We suggest that lattice studies at high isospin chemical potential in the vector channel for QCD might be able to, indirectly, shed light on this sector of the theory at zero chemical potential. More generally the hope is that these studies might help understanding how to construct consistent theories of interacting massive higher spin fields not necessarily related to a gauge principle.

The present knowledge can be used for a number of physical phenomena of topical interest. For example in the framework of 2 color QCD at non zero baryon chemical potential[25, 26] vector condensation has been predicted in [17, 16]. Recent lattice studies [18] seem to support it. Studies [27] of the Gross-Neveu model in $2+1$ dimensions with a baryon chemical potential might also shed light on the vector meson channel. The present analysis while reinforcing the scenario of vector condensation shows that we can have many different types of condensations with very distinct signatures. Other possible physical applications are discussed in [16]. The analysis has been extended to a general number of space dimensions [16] and is useful for various scenarios related to the phenomenon of vector condensation [8, 28]. 


\section{REFERENCES}

1. A. D. Linde, Phys. Lett. B 86, 39 (1979).

2. E. J. Ferrer, V. de la Incera and A. E. Shabad, Phys. Lett. B 185, 407 (1987).

3. E. J. Ferrer, V. de la Incera and A. E. Shabad, Nucl. Phys. B 309, 120 (1988).

4. J. Ambjorn and P. Olesen, Phys. Lett. B 218, 67 (1989) [Erratum-ibid. B 220, 659 (1989)], ibid. B 257, 201 (1991), Nucl. Phys. B 330, 193 (1990).

5. K. Kajantie, M. Laine, J. Peisa, K. Rummukainen and M. E. Shaposhnikov, Nucl. Phys. B 544, 357 (1999) |arXiv:hep-lat/9809004|.

6. N. S. Manton, Nucl. Phys. B 158, 141 (1979).

7. Y. Hosotani, Annals Phys. 190, 233 (1989).

8. L. F. Li, arXiv:hep-ph/0210063

9. G. E. Brown and M. Rho, Phys. Rev. Lett. 66, 2720 (1991). T. Hatsuda and S.H. Lee, Phys. Rev. bf C46 (1992) 34.

10. K. Langfeld, H. Reinhardt and M. Rho, Nucl. Phys. A662 (1997) 620; K. Langfeld, Nucl. Phys. A642 $96 \mathrm{c}$.

11. M. Harada and K. Yamawaki, Phys. Rev. Lett. 86, 757 (2001) arXiv:hep-ph/0010207|.

12. M. Bando, T. Kugo and K. Yamawaki, Phys. Rept. 164, 217 (1988).

13. F. Sannino and K. Tuominen, arXiv:hep-ph/0303167 To Appear in PRD.

14. F. Sannino and K. Tuominen, arXiv:hep-ph/0305004

15. F. Sannino, Phys. Rev. D 67, 054006 (2003) |arXiv:hep-ph/0211367|.

16. F. Sannino and W. Schafer, Phys. Lett. B 527, 142 (2002) |arXiv:hep-ph/0111098|.

17. J. T. Lenaghan, F. Sannino and K. Splittorff, Phys. Rev. D 65, 054002 (2002) |arXiv:hep-ph/0107099|.

18. B. Alles, M. D'Elia, M. P. Lombardo and M. Pepe, arXiv:hep-lat/0210039 (See references therein for 2 color QCD at high baryon chemical potential)

19. S. Muroya, A. Nakamura and C. Nonaka, arXiv:hep-lat/0211010

20. T. Appelquist, P.S. Rodrigues da Silva and F. Sannino, Phys. Rev. D60, 116007 (1999), hep-ph/9906555

21. Z. Duan, P.S. Rodrigues da Silva and F. Sannino, Nucl. Phys. B 592, 371 (2001), hep-ph/0001303

22. T. Schafer, D. T. Son, M. A. Stephanov, D. Toublan and J. J. Verbaarschot, hep-ph/0108210 V. A. Miransky and I. A. Shovkovy, hep-ph/0108178

23. H.B. Nielsen and S. Chadha, Nucl. Phys. B105, 445 (1976).

24. J. I. Kapusta, Finite-temperature field theory, Cambridge Monographs on Mathematical Physics (1993).

25. K. Splittorff, D. Toublan and J. J. Verbaarschot, Nucl. Phys. B 639, 524 (2002) |arXiv:hep-ph/0204076|. See also references therein.

26. G. V. Dunne and S. M. Nishigaki, arXiv:hep-ph/0306220

27. S. Hands, J. B. Kogut, C. G. Strouthos and T. N. Tran, arXiv:hep-lat/0302021

28. J. W. Moffat, arXiv:hep-th/0211167 